\title{
Molecular characterization of immune responses of Helicoverpa armigera to infection with the mermithid nematode Ovomermis sinensis
}

\author{
Gui-Jie Wang, Xiao-Rong Zhuo, Wen-Wen Wang, Xu-Sheng Liu, Guo-Xiu Wang and Jia-Lin Wang* (D)
}

\begin{abstract}
Background: Mermithid nematodes, such as Ovomermis sinensis, display a broad host range including some lepidopteran pests. Infective juveniles penetrate their host through the cuticle, complete their growth within the hemocoel and eventually kill the host upon their emergence. Hence, mermithid nematodes are considered potential biological control agents of insect pests. Our previous data indicate that the infection rate of $O$. sinensis on cotton bollworm (Helicoverpa armigera) is low, which may be largely due to the strong immune system of the host. However, current knowledge on the interactions of mermithid nematodes with their hosts and the mechanisms employed by hosts to defend themselves against mermithid nematodes is limited.

Results: Here, we investigated the response of $H$. armigera to $O$. sinensis infection. Parasitism by $O$. sinensis caused a sharp decline in the survival rate of $H$. armigera. The hemocytic phagocytosis ability, antibacterial activity, and phenoloxidase (PO) activity in plasma of $\mathrm{H}$. armigera increased at $1 \mathrm{~d}$ post parasitism (dpp) but decreased at $3 \mathrm{dpp}$. Further, we investigated gene expression in the fat body of parasitized and non-parasitized $H$. armigera larvae at 1 , 3 , and 5 dpp using a digital gene expression system. In total, 41, 60 and 68 immune-related differentially expressed genes were identified at 1, 3, and 5 dpp, respectively. These genes encoded pattern recognition receptors (PRRs), antimicrobial peptides (AMPs), serine proteases (SPs), SP inhibitors, mucins and other immune-related proteins. The expression of most PRRS, AMPS, SPS, and mucins was upregulated in the fat body of larvae at $1 \mathrm{dpp}$, downregulated at $3 \mathrm{dpp}$, and then again upregulated at $5 \mathrm{dpp}$ by $\mathrm{O}$. sinensis. The increased expression of SP inhibitors may contribute to the inhibited PO activity at $5 \mathrm{dpp}$.
\end{abstract}

Conclusions: This study demonstrates that parasitism by $O$. sinensis modulates the immune reaction of the host $H$. armigera by altering the expression of immune-related genes. Our data provide a basis for future investigation of the molecular mechanisms employed by the mermithid nematode $O$. sinensis to modulate the immunity of the host $H$. armigera. These data will also likely facilitate the improvement of success in parasitism of $\mathrm{H}$. armigera by $\mathrm{O}$. sinensis.

Keywords: Ovomermis sinensis, Helicoverpa armigera, Immunity, Parasitism, Fat body

\footnotetext{
* Correspondence: jlwang@mail.ccnu.edu.cn

Hubei Key Laboratory of Genetic Regulation and Integrative Biology, School

of Life Sciences, Central China Normal University, Wuhan 430079, China
}

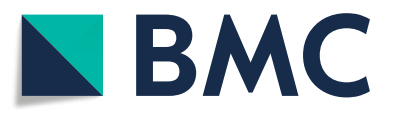

(c) The Author(s). 2019 Open Access This article is distributed under the terms of the Creative Commons Attribution 4.0 International License (http://creativecommons.org/licenses/by/4.0/), which permits unrestricted use, distribution, and reproduction in any medium, provided you give appropriate credit to the original author(s) and the source, provide a link to the Creative Commons license, and indicate if changes were made. The Creative Commons Public Domain Dedication waiver (http://creativecommons.org/publicdomain/zero/1.0/) applies to the data made available in this article, unless otherwise stated. 


\section{Background}

Insects are one of the most successful species on earth. They occupy diverse habitats and survive under different biotic and abiotic stresses. To combat microbial or parasitic infections, insects have evolved cellular and humoral immune responses, which are initiated rapidly following the recognition of pathogens by pattern recognition receptors (PRRs). Cellular immune responses involve hemocyte-mediated encapsulation, phagocytosis, and nodulation, whereas humoral immune responses mainly involve the induction of antimicrobial peptides (AMPs) and prophenoloxidase (PPO) activating system $[1,2]$.

Entomopathogenic nematodes (EPNs) naturally infect and kill insects, thus representing a crucial alternative to chemicals for pest control $[3,4]$. Nematodes employ different strategies to survive within the host insect, either by evading or suppressing the host immune response. EPNs belonging to the families Heterorhabditidae and Steinernematidae are well studied and widely used for pest control $[3,5]$. Infective juveniles of the nematodes release symbiotic bacteria into the hemocoel after entering the insect host, thus killing the host within 24 to 48 h. Virulence factors such as toxin complexes, lipases, proteases, and lipopolysaccharides produced by symbiotic bacteria suppress host immune response $[6,7]$.

Despite impressive advances in the immune response of insects to microbial infection, our understanding of the molecular mechanisms involved in host-nematode interactions and host antiparasitic immune reactions remains obscure. Some studies suggest that nematodes modulate the host immune system via symbiotic bacteria. For example, Heterorhabditid nematodes are either not recognized or less efficiently recognized by the host immune system, and the induced PRRs of the host are response to the symbiotic bacteria of nematodes [8]. Similarly, the transcription of several AMP genes is activated in Manduca and Drosophila larvae upon infection with Heterorhabditis associated with the symbiotic bacteria Photorhabdus but not upon infection with axenic worms lacking the symbiotic bacteria $[8,9]$. Other studies indicate that nematodes alone alter the transcription of certain immune-related genes. For example, infection of axenic Heterorhabditis nematodes results in the induction of several immune-related genes in adult flies [10]. Transcripts of several PRRs, serine proteases (SPS), SP inhibitors (serpins), and AMPs vary in Armigeres subalbatus mosquitoes infected with the filarial parasite Brugia malayi [11].

The developing stages of mermithid nematodes are parasitic, whereas the adults are free-living. Infective juveniles parasitize their host by penetrating through the cuticle. Once parasitism is established, the juveniles complete their growth inside the host, typically reaching impressive sizes. The juveniles eventually kill the host upon their emergence, suggesting the potential of mermithids for the biocontrol of insect pests. Ovomermis sinensis, a mermithid nematode, has been reported to display a broad host range, including lepidopteran pests such as the cotton bollworm (Helicoverpa armigera) [12]. Li et al. (2009) have demonstrated that parasitism with $O$. sinensis changes the spreading behavior of hemocytes and suppresses hemocytic encapsulation abilities of $H$. armigera [13]. We have previously shown that $H$. armigera C-type lectin 3 (CTL3) binds to the surface of O. sinensis and contributes to antiparasitic immune response [14].

However, the interactions of mermithid nematodes with their hosts and the immune response of the host to infection are poorly understood. In the present study, we evaluated the effect of parasitism by $O$. sinensis on the survival, phagocytosis ability, antibacterial activity, and PO activity of $H$. armigera. Further, gene expression dynamics in the fat body of parasitized and non-parasitized $H$. armigera larvae were investigated, and differentially expressed immune-related genes were obtained. These data improve our understanding of host-nematode interactions, and provide a comprehensive resource for exploring the molecular mechanism employed by the mermithid nematode $O$. sinensis to modulate the immune system of $H$. armigera.

\section{Results}

Effect of parasitism by $\mathrm{O}$. sinensis on $\mathrm{H}$. armigera survival

To evaluate the effect of $O$. sinensis on the pest control, the survival rate of $H$. armigera larvae parasitized by $O$. sinensis was investigated. The survival rate of parasitized larvae was much lower than that of the mock group, and all infected larvae died within $13 \mathrm{~d}$ post parasitism (dpp) (Fig. 1A). Juveniles of O. sinensis initially got fully developed at $9 \mathrm{dpp}$ and emerged through the integument of the host, thereby killing the larvae (Fig. 1B). During the emergence of $O$. sinensis from 9 to $13 \mathrm{dpp}$, the parasitized group of $H$. armigera remained sixth-instar larvae while the mock group was pupae.

\section{Effect of parasitism by $O$. sinensis on $H$. armigera immune response}

To test whether $O$. sinensis modulates the host immune response, we compared phagocytosis, antibacterial activity, and PO activity in plasma between parasitized and non-parasitized $H$. armigera larvae. The results showed that hemocytic phagocytosis of Escherichia coli was enhanced at $1 \mathrm{dpp}$ and then decreased at $3 \mathrm{dpp}$, with no significant differences at $5 \mathrm{dpp}$ (Fig. 2A and B). Antibacterial activities in the plasma were promoted at 1 and $5 \mathrm{dpp}$ and reduced at $3 \mathrm{dpp}$ (Fig. 2C). Plasma PO activity was slightly increased at $1 \mathrm{dpp}$, and then significantly reduced at 3 and $5 \mathrm{dpp}$ (Fig. 2D). Since parasitism by O. sinensis enhanced phagocytosis and antibacterial activity in host at $1 \mathrm{dpp}$, we wonder whether $O$. sinensis was associated with bacteria. PCR analyses indicated that $O$. sinensis juveniles harbored bacteria (Additional file 1: Figure S1). 
A - Mock - O. sinensis infected

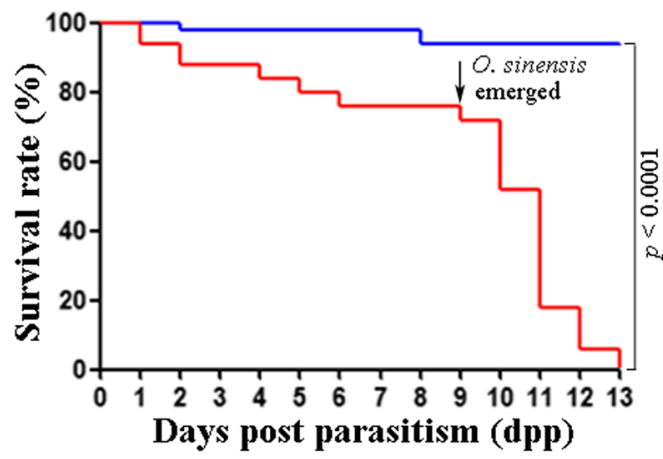

B
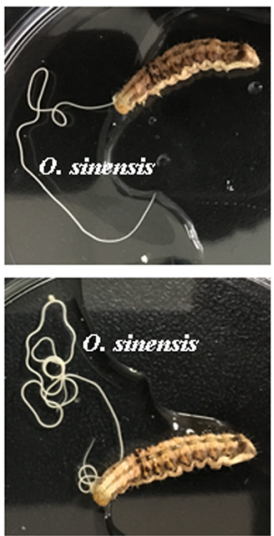

Fig. 1 Parasitism by Ovomermis sinensis decreased Helicoverpa armigera survival. (a) Survival curves of $H$. armigera larvae infected with $O$. sinensis (red line; $n=50$ ) in comparison with non-parasitized larvae (control; blue line; $n=50$ ). O. sinensis emergence started at $9 \mathrm{~d}$ post parasitism (dpp), causing a sharp decline in the survival rate of $H$. armigera larvae. Significant difference $(p<0.0001$, Log-rank test) was observed between 0 . sinensis-infected larvae and control groups. (b) O. sinensis emerged partially (upper panel) or completely (lower panel) from $\mathrm{H}$. armigera larva

\section{RNA-Seq analysis and identification of differentially expressed transcripts (DETs)}

To explore the molecular mechanism employed by $O$. sinensis to alter host immunity, we compared digital gene expression (DGE) profiles in the fat body of parasitized (FP) and non-parasitized (control; FC) H. armigera larvae.
FP and FC were dissected from larvae at 1 (FP1, FC1), 3 (FP3, FC3), and 5 (FP5, FC5) dpp, and three biological replicates were performed at each time point. Parasitism by $O$. sinensis delays $H$. armigera larval development. FP1 and $\mathrm{FC} 1$ represent fat bodies both of fifth-instar larvae at feeding stage. FP3 and FC3 correspond to fat bodies of
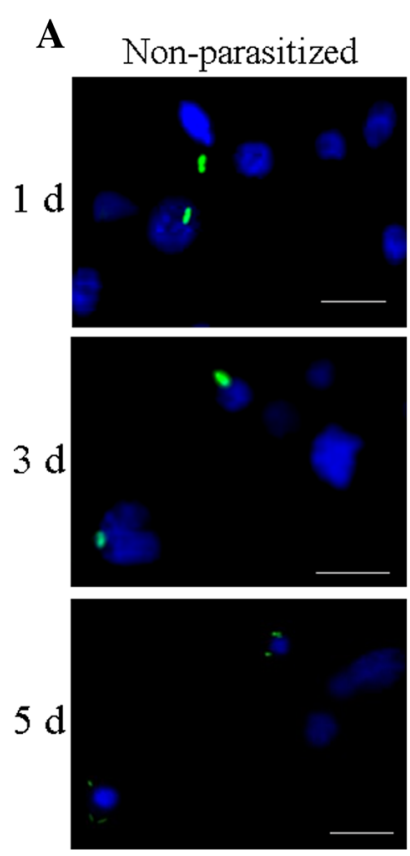
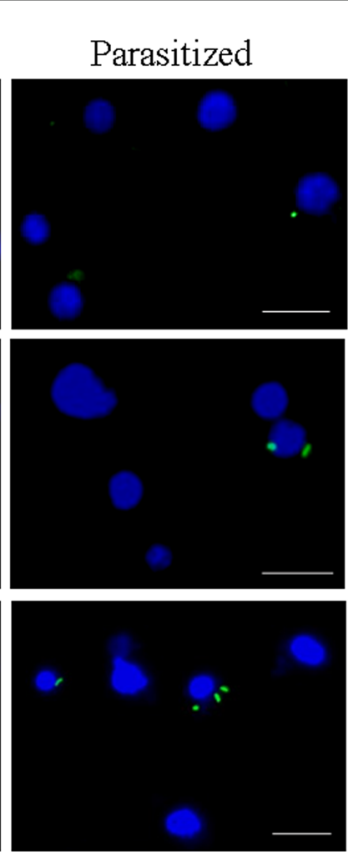

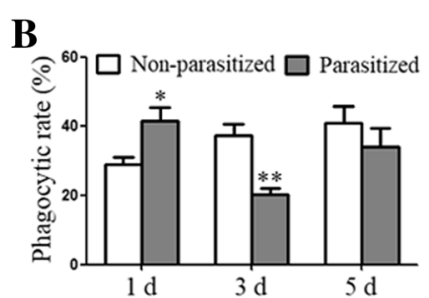

C

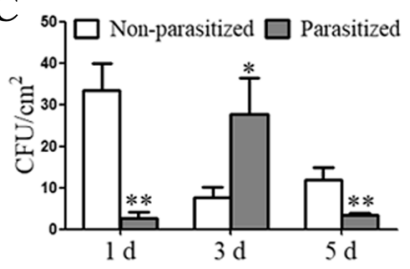

D

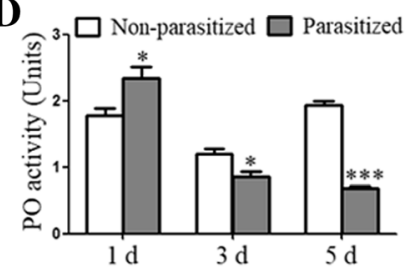

Fig. 2 Dynamic immune responses of $H$. armigera larvae infected with $O$. sinensis. (a) Representative images of phagocytosis of Escherichia coli. (b) Statistical analysis of phagocytic rate. Hemocytes were collected from parasitized and non-parasitized H. armigera larvae at 1, 3, and 5 dpp. FITC-labeled E. coli (green) were subjected to phagocytosis analyses. DAPI was used to label hemocytic nucleus (blue). Scale bar $=10 \mu \mathrm{m}$. (c, d) Changes in antibacterial activity (c) and PO activity (d) in the plasma of H. armigera larvae. Plasma were collected from parasitized and non-parasitized larvae at 1, 3 and $5 \mathrm{dpp}$. Data represent mean \pm standard error of mean (SEM) for three biological replicates. Significant differences were determined using Student' $\mathrm{s} t$-test and are indicated with asterisks $\left({ }^{*} 0.01<p<0.05,{ }^{* *} 0.001<p<0.01,{ }^{* * *} p<0.001\right)$ 
fifth-instar larvae at head capsule slippage (HCS) and sixth-instar larvae at feeding stage, respectively. FP5 and FC5 represent fat bodies of sixth-instar larvae at feeding stage and metamorphic stage, respectively. In total, 18 RNA-Seq libraries (FP1-1, - 2, - 3; FC1-1, - 2, - 3; FP31, - 2, - 3; FC3-1, - 2, - 3; FP5-1, - 2, - 3; FC5-1, - 2, 3 ) were constructed and sequenced. The number of raw reads generated from each library ranged from 23.96 to 27.92 million, whereas the number of cleaned reads ranged from 21.01 to 23.62 million. Cleaned reads from each library were mapped to previously assembled unigenes of $H$. armigera fat body [15], with a mapping ratio ranging from 49.36 to $58.76 \%$ (average $52.91 \%$ ) (Additional file 2: Table S1).

To compare the differential gene expression profiles between FP and FC larvae at different time points (FP1 vs. FC1; FP3 vs. FC3; FP5 vs. FC5), fragments per kilobase of transcript per million mapped fragments (FPKM) were quantified. Heatmap indicated that the square of correlation coefficient ranged from 0.909-0.99, 0.975-0.993, 0.994-0.996, 0.871-0.977, 0.978-0.99, and 0.912-0.99 in FC1, FC3, FC5, FP1, FP3, and FP5 groups, respectively (Additional file 3: Figure S2). These high Pearson values suggest the reliable replications and the reasonable samples.

NOISeq-sim was adopted to screen DETs with cut-off thresholds of $\mid \log 2$ (FP/FC ratio) $\mid \geq 1$ and divergent probability $\geq 0.8$. A total of $1687 \mathrm{DETs}$ were identified from the FP1 vs. FC1, of which 647 were upregulated and 1040 were downregulated. A total of 1352 DETs were upregulated and 1098 DETs were downregulated from the FP3 vs. FC3, whereas 5459 DETs were upregulated and 2221 DETs were downregulated from the FP5 vs. FC5 (Fig. 3 and Additional file 4: Table S2).
Expression dynamics of immune-related genes in the parasitized fat body

Of the 1687 DETs identified from the FP1 vs. FC1 comparison, $80 \mathrm{DETs}$ representing 41 genes were involved in immunity; these encoded 11 PRRs, 4 AMPs, 12 SPs, 3 SP inhibitors, 6 mucins and 5 others. Of the 2450 DETs identified from the FP3 vs. FC3 comparison, 131 DETs representing 60 genes were involved in immunity, including 16 PRRs, 12 AMPs, 14 SPs, 4 SP inhibitors, 6 mucins and 8 others. Of the 7680 DETs identified from the FP5 vs. FC5 comparison, 209 DETs representing 68 genes were involved in immunity, including 15 PRRs, 10 AMPs, 12 SPs, 11 SP inhibitors, 6 mucins, and 14 others (Fig. 4 and Additional file 5: Table S3).

Most of the PRR, AMP, SP, and mucin genes were upregulated in parasitized larvae at $1 \mathrm{dpp}$, downregulated at $3 \mathrm{dpp}$ and again upregulated at $5 \mathrm{dpp}$, compared with non-parasitized larvae. Two PRRs (CTL4, CTL7), two AMPs (gloverin, cecropin 3), two SPs (SP4, azurocidin-like $S P$ ), and two mucins (mucin protein, mucin 4), which were shared among FP1 vs. FC1, FP3 vs. FC3, and FP5 vs. FC5 groups, were selected for quantitative real-time PCR (qRT-PCR) analyses. The results of qRT-PCR validated RNA-Seq results (Fig. 5). The expression of most SP inhibitors (serpins) was upregulated in parasitized larvae at 5 dpp, compared with non-parasitized larvae.

\section{Expression of $P R R$ genes}

Four differentially expressed PRRs [Gram negative binding protein $(G N B P)$ precursor, peptidoglycan recognition protein $B$ (PGRP B), PGRP C, and PGRP D] were shared between FP1 vs. FC1 and FP3 vs. FC3 comparisons, all of which were upregulated in FP1 vs. FC1 and downregulated in FP3

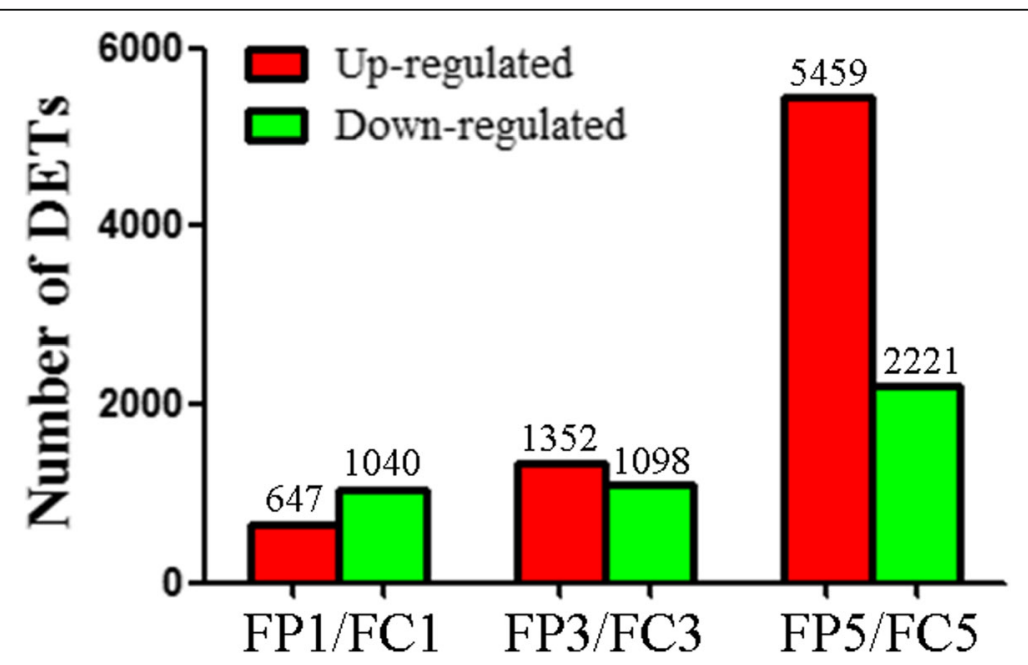

Fig. 3 Comparisons of differentially expressed transcripts (DETs) between the FP1 and FC1, FP3 and FC3, and FP5 and FC5. The FP1, FP3, and FP5 represent the fat body of $\mathrm{H}$. armigera larvae parasitized by $\mathrm{O}$. sinensis at 1,3 , and $5 \mathrm{dpp}$, respectively. The FC1, FC3, and FC5 represent the fat body of non-parasitized larvae (controls) collected at the same time points. The number of upregulated DETs is indicated in the red column, whereas the number of downregulated DETs is represented in green 

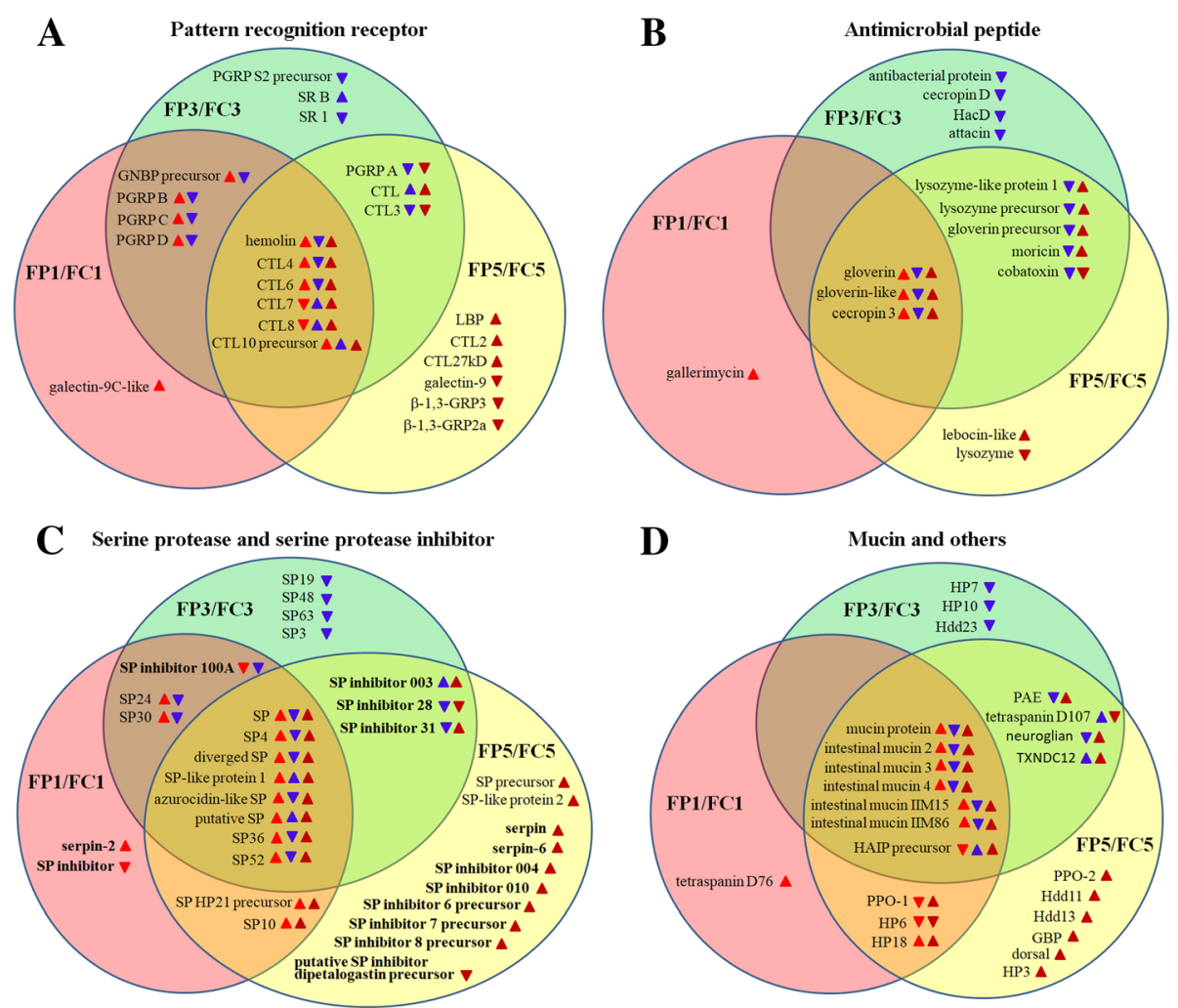

Fig. 4 Schematic representation of common and unique immune-related differentially expressed genes identified based on comparisons between FP and FC samples. (a) Pattern recognition receptors (PRRs). (b) Antimicrobial peptides (AMPS). (c) Serine proteases (SPS) and SP inhibitors (serpins). (d) Mucins and others. The FP1, FP3, and FP5 represent the fat body of $\mathrm{H}$. armigera larvae parasitized by $\mathrm{O}$. sinensis at 1, 3, and $5 \mathrm{dpp}$, respectively, whereas FC1, FC3, and FC5 represent the fat body of non-parasitized larvae (controls) at the same time points. Genes in overlapping regions were identified as differentially expressed in two or three groups. Red, blue, and purple arrows represent expression level changes in FP1 vs. FC1, FP3 vs. FC3, and FP5 vs. FC5, respectively. Upward- and downward-pointing arrows represent the directions of expression level changes

vs. FC3. The PGRP $A$ and $C T L 3$ genes were downregulated, whereas CTL was upregulated in both FP3 vs. FC3 and FP5 vs. FC5 comparisons. No overlap in the PRRs that showed differentially expressed between FP1 vs. FC1 and FP5 vs. FC5. Additionally, six differentially expressed PRRs (hemolin, CTL4, CTL6, CTL7, CTL8, and CTL10 precursor) were shared among FP1 vs. FC1, FP3 vs. FC3, and FP5 vs. FC5; the expression of hemolin, CTL4 and CTL6 was first increased in FP1 vs. FC1, decreased in FP3 vs. FC3, and then again increased in FP5 vs. FC5 (Fig. 4A).

\section{Expression of $A M P$ genes}

Five differentially expressed AMPs (lysozyme-like protein 1, lysozyme precursor, gloverin precursor, moricin, and cobatoxin) were shared between FP3 vs. FC3 and FP5 vs. FC5. No overlap in the $A M P$ transcripts that showed differentially expressed either between FP1 vs. FC1 and FP3 vs. FC3 or between FP1 vs. FC1 and FP5 vs. FC5. Of the differentially expressed AMP genes, three (gloverin, gloverin-like and cecropin 3) were shared among FP1 vs. FC1, FP3 vs. FC3, and FP5 vs. FC5. All of $A M P$ genes identified were upregulated in FP1 vs. FC1, downregulated in FP3 vs. FC3 and upregulated in FP5 vs. FC5, with the exception of cobatoxin and lysozyme, which were downregulated in FP5 vs. FC5 (Fig. 4B).

\section{Expression of $S P$ and $S P$ inhibitor genes}

Two differentially expressed $S P$ genes $(S P 24, S P 30)$ and one differentially expressed $S P$ inhibitor gene (SP inhibitor $100 A$ ) were shared between FP1 vs. FC1 and FP3 vs. FC3. Two additional differentially expressed SPS (SP HP21 precursor, SP10) were shared between FP1 vs. FC1 and FP5 vs. FC5, and three differentially expressed $S P$ inhibitors (SP inhibitor 003, 28, and 31) were shared between FP3 vs. FC3 and FP5 vs. FC5. Among the differentially expressed $S P$ genes, eight $(S P, S P 4$, diverged SP, SP-like protein 1, azurocidin-like SP, putative SP, SP36, and SP52) were shared among FP1 vs. FC1, FP3 vs. FC3, and FP5 vs. FC5; the expression of all of these eight genes was upregulated in FP1 vs. FC1 and FP5 vs. FC5, whereas downregulated in FP3 vs. FC3 with the exception of SP-like protein 1 and putative $S P$, which were upregulated. Additionally, nine SP inhibitor genes (serpin, serpin-6, SP inhibitor 003, 

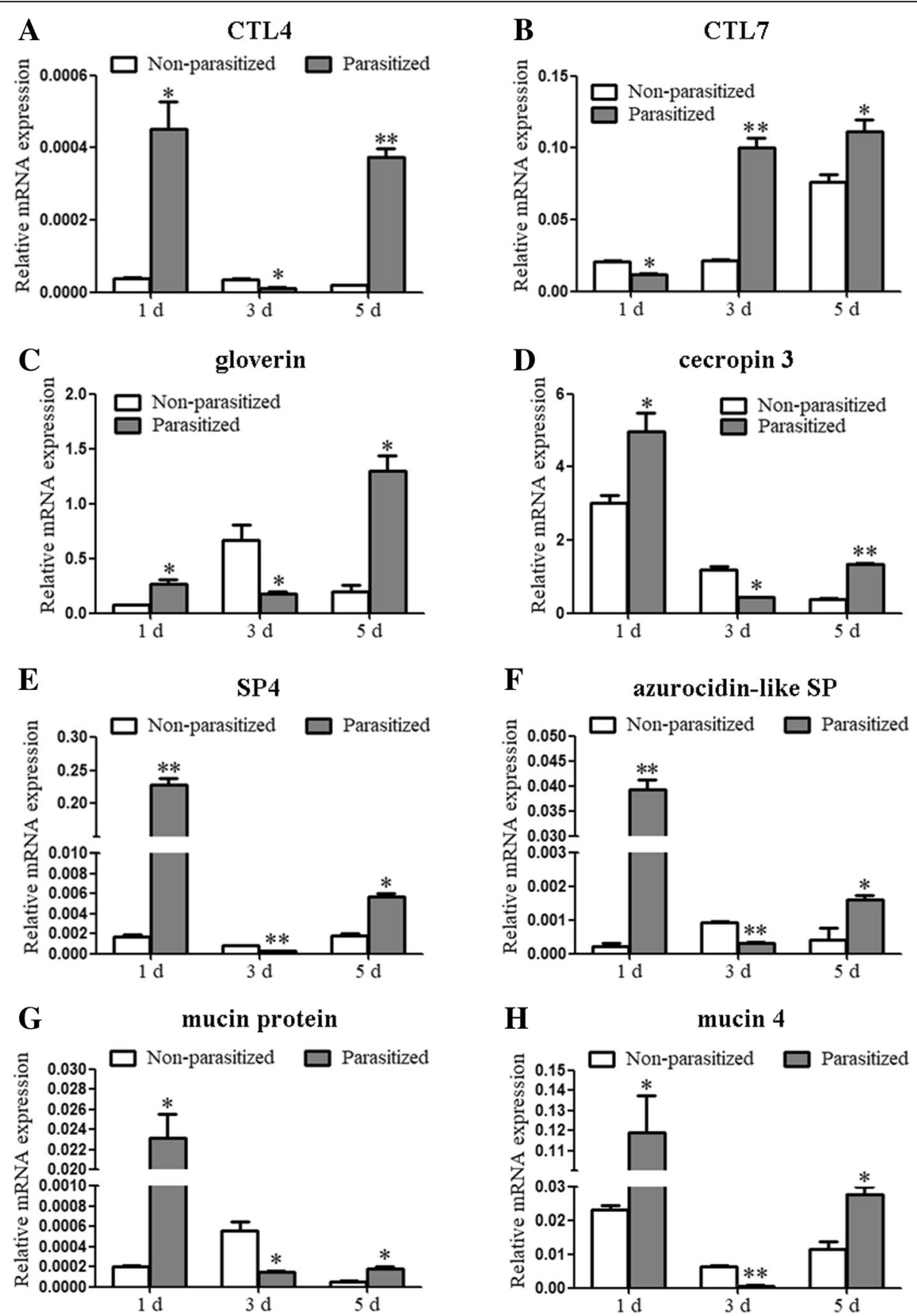

Fig. 5 Validation of RNA-Seq data using qRT-PCR analysis. a CTL4. b CTL7. c gloverin. $\mathbf{d}$ cecropin 3. e SP4. $\mathbf{f}$ azurocidin-like SP. $\mathbf{g}$ mucin protein. $\mathbf{h}$ mucin 4. Fat bodies were dissected from parasitized and non-parasitized H. armigera larvae, and the expression level of several immune-related genes was analyzed using qRT-PCR. All expression values are calculated against the reference gene $\beta$-actin. Data represent mean \pm SEM of three biological replicates. Significant differences were determined using Student's $t$-test and are indicated using asterisks $\left({ }^{*} 0.01<p<0.05\right.$, ** $0.001<p<0.01)$

004, 010, 6 precursor, 7 precursor, 8 precursor and 31) were upregulated in FP5 vs. FC5 (Fig. 4C).

\section{Expression of mucin and other genes}

Six mucin genes [mucin protein, intestinal mucin (IIM) 2, 3, 4, IIM15, and IIM86] were upregulated in FP1 vs.
FC1, downregulated in FP3 vs. FC3, and again upregulated in FP5 vs. FC5. The abundance of PPO-1 transcripts was inhibited in FP1 vs. FC1, whereas transcripts of prophenoloxidase activating enzyme (PAE) were suppressed in FP3 vs. FC3. The PPO-1, PPO-2, and PAE transcripts were increased in FP5 vs. FC5 (Fig. 4D). 


\section{Discussion}

Unlike EPNs (Heterorhabditidae and Steinernematidae) that kill their host within $24-48 \mathrm{~h}$ post infection, the mermithid nematode $O$. sinensis killed most of the $H$. armigera larvae within 9-13 dpp in this study. The efficient insecticidal effect of Heterorhabditidae and Steinernematidae is due to their symbiotic bacteria, which are released within the insect hemocoel and secrete virulence factors [6, 7]. Hence, this nematode-bacteria symbiosis is crucial for efficient action of EPNs in pest control [16]. The mermithid nematode Strelkovimermis spiculatus serves as a vector of the iridescent virus [17]; however, association of mermithid nematode with symbiotic bacteria has been rarely detected [18]. Although $O$. sinensis was associated with bacteria, we cannot conclude whether the bacteria was symbiotic or came from outside contamination. Considering that $O$. sinensis kills the host mostly by emerging through the host integument, which takes a relatively long time, it is likely that O. sinensis does not harbor pathogenic bacteria.

O. sinensis not only takes a long time to kill its hosts, but also exhibits a relatively low infection rate in the host $H$. armigera, as shown in our laboratory [14]. The low infection rate of $O$. sinensis on $H$. armigera may be largely due to the highly developed immune system of the host. Hence, clarification of the interactions between $O$. sinensis and its host, especially the immune response of $H$. armigera to $O$. sinensis is of vital importance. The relatively low mapping ratio of the cleaned reads to previously assembled unigenes of peptidoglycan (PGN)-challenged fat body was observed. This may reflect the differences in the transcriptional profiles between the $O$. sinensis-challenged fat body and the PGN-challenged one, or the insufficient sequencing depth of the previous fat body transcriptome [15]. The number of DETs between FP and FC increased along with larval development. These DETs represent a broad range of pathways, including metabolic pathways, fat digestion and absorption, fatty acid biosynthesis, steroid biosynthesis and Ribosome (data not shown). Here, we focused on molecular characterization of immune responses.

Insects employ hemocytic phagocytosis against bacteria, which requires the participation and coordination of both cellular and humoral factors. Several humoral PRRs such as opsonic factors, secreted mainly from fat body, function in non-self recognition and triggering phagocytosis [19, 20]. Since the phagocytosis ability of $H$. armigera is largely due to PRRs, which attach to hemocytes, variation in the expression levels of genes encoding PRRs at different time points post parasitism by $O$. sinensis would have important consequences. The expression of many $P R R$ genes is consistent with the phagocytosis ability of $H$. armigera, as some PRR proteins are involved in phagocytosis. For example, PGRP D has previously been demonstrated to promote phagocytosis of E. coli in $H$. armigera [21]. In this study, the upregulation and downregulation of PGRP D expression at 1 and $3 \mathrm{dpp}$ by $O$. sinensis, respectively, were consistent with the phagocytosis ability of $H$. armigera at these time points. Similar dynamic expression of PRRs has also been detected in $A$. subalbatus in response to $B$. malayi infection, with a PGRP transcript upregulated at 1 h post infection and a CTL transcript downregulated at 12 or $24 \mathrm{~h}$ post infection [11].

The EPN Steinernema feltiae modulates hemocytic phagocytosis by removing opsonic factors from the host hemolymph, thus supporting its symbiotic bacteria [22]. Since O. sinensis most likely does not need to support symbiotic bacteria, initial infection of $O$. sinensis is presumed to activate the host immune system, for example by activating phagocytosis. In addition to the role of PRRs in phagocytosis, some PRRs also participate in encapsulation. For example, infection of $A$. subalbatus with the filarial worm Dirofilaria immitis leads to induction of $\beta 1,3$-glucan recognition protein, a PRR involved in encapsulation [23]. Although the expression of most $P R R$ genes was upregulated in $H$. armigera at $1 \mathrm{dpp}$ by $O$. sinensis, encapsulation was suppressed possibly because of the destruction of hemocyte cytoskeleton, as demonstrated previously [13]. Parasitization also suppresses host cellular encapsulation by inhibiting the expression of PRRs such as CTL and scavenger receptor $[24,25]$. Given that PGRP D and CTL3 have been previously demonstrated to promote encapsulation $[14,21]$, we speculate that the decreased expression of certain PRRs at $3 \mathrm{dpp}$ would facilitate the survival of $O$. sinensis within the homocoel of $H$. armigera.

Antimicrobial effectors such as AMPs are mainly synthesized in fat body and subsequently secreted into the hemolymph, and play important roles in the restriction or elimination of the invading pathogen [26]. Infection of Aedes aegypti with Wuchereria bancrofti filarial nematodes has been shown to increase mRNA levels of defensin, cecropin and transferrin [27]. Some researchers argue that the upregulation of $A M P$ transcripts may be attributed to symbiotic bacteria, considering it is symbiotic nematodes but not axenic nematodes that induced the transcription of $A M P$ genes [8,9]. Other studies have raised the possibility that the increased expression of immune-related genes (including $A M P S$ ) reflects strategies for tolerating tissue damage caused by nematodes [28]. Cecropin was reported to attenuate the motility of the filarial nematode Brugia pahangi in vitro and reduce the number of $B$. pahangi in vivo [29]. Hence, we speculate that the expression of $A M P$ genes is increased in response to either $O$. sinensis or bacteria associated with $O$. sinensis, which needs further investigation.

To establish infection, nematodes must be capable of suppressing the immunity of their host. Nematodes modulate the humoral and cellular immune responses of 
the host by producing molecules such as proteases. For example, symbiotic Heterorhabditis bacteriophora secrete a proteinase into the bodies of greater wax moth larvae and inhibit the expression of cecropin [30]. In this study, we showed upregulation of $A M P$ genes in $H$. armigera at $1 \mathrm{dpp}$ by $O$. sinensis and downregulation at $3 \mathrm{dpp}$; this was consistent with the antibacterial activity in plasma. The $A M P$ genes exhibit similar expression profiles in A. subalbatus at different time points post infection by filarial worm. For example, the expression of two cecropin genes is increased at $6 \mathrm{~h}$ post infection and that of two defensin and two lysozyme genes is decreased at $12 \mathrm{~h}$ post infection [11]. Since molecules produced by $O$. sinensis have not yet been characterized, the mechanisms employed by $O$. sinensis to suppress the immunity of the host remain unclear. Interestingly, we observed upregulation of $A M P$ genes and enhanced antibacterial activity in $H$. armigera larvae at $5 \mathrm{dpp}$ by $O$. sinensis, possibly because $O$. sinensis may have developed sufficiently during $5 \mathrm{~d}$ to endure higher immune stress. Mucin is reported to be involved in the entrapment of bacteria [31]. In this study, mucin genes exhibited a similar expression profile as the $A M P$ genes; thus, it is likely that mucins function in antiparasitic immunity and contribute toward antibacterial activity in plasma.

SPs and SP inhibitors (serpins) play important roles in melanin biosynthesis, and are most likely involved in antiparasitic immunity [32, 33]. Global transcriptional response of $A$. aegypti to $B$. malayi infection has revealed an increase in the abundance of several $S P$ transcripts [28]. Transcriptional levels of $30 S P$ or serpin genes vary in $A$. subalbatus at different time points following infection by B. malayi [11], suggesting a potential role of melanization in antiparasitic immunity. In this study, the abundance of most $S P$ transcripts in $H$. armigera larvae parasitized by $O$. sinensis increased at $1 \mathrm{dpp}$ and decreased at $3 \mathrm{dpp}$. Given that SP functions in the activation of PPO and is required for parasite melanization [34, 35], it is reasonable that PO activity in plasma slightly increased at $1 \mathrm{dpp}$ and decreased at $3 \mathrm{dpp}$. However, at $5 \mathrm{dpp}$, the abundance of most of the $S P$ transcripts increased, whereas PO activity decreased. This may be because of the increased abundance of SP inhibitor transcripts, as serpin inhibits the activation of PPO by SP [36].

Some virulence factors produced by parasites during infection protect them against the potent effects of the immune system of the host and improve the success rate of parasitism. For example, a trypsin-like serine protease and a chymotrypsin serine protease secreted during the parasitic phase of Steinernema carpocapsae exhibit PPO inhibitory activity [37, 38]. Here we showed that $O$. sinensis parasitism modulated $\mathrm{PO}$ activity of $H$. armigera larvae by altering the expression of SPS and serpins, although the underlying mechanisms remain unclear.

\section{Conclusions}

Overall, our data revealed dynamic immune responses of $H$. armigera to $O$. sinensis infection. The initial infection of $H$. armigera by $O$. sinensis activated the expression of many PRR, AMP, SP, and mucin genes, which is consistent with the enhanced immune reactions of the host (phagocytosis, antibacterial activity, and PO activity). Subsequently, $O$. sinensis suppressed these immune reactions by inhibiting the expression of most PRR, AMP, SP, and mucin genes to facilitate its survival within the host. After the successful establishment of $O$. sinensis parasitism, we speculate that $O$. sinensis was able to endure the relatively higher immune-related stress (such as antibacterial activity) presumably because it has grown to a sufficiently large size within $5 \mathrm{dpp}$. However, the inhibited PO activity at 5 dpp may be attributed to the increased expression of serpins, suggesting that $O$. sinensis is more sensitive to melanization. We provide a comprehensive resource for exploring the complex molecular mechanisms underlying the interaction between the mermithid nematode $O$. sinensis and its host $H$. armigera. Further investigation of DEGs between FP and FC would provide critical target genes for improvement of infection rate. Characterization of virulence factors produced by $O$. sinensis and elucidation of the mechanism employed by $O$. sinensis to suppress host immunity will also be our future study. Our findings will likely facilitate the development of $O$. sinensis as an effective and eco-friendly biological control agent.

\section{Methods}

\section{Culture conditions, PCR amplification and infection of $H$. armigera larvae}

$H$. armigera larvae were maintained in the laboratory at $28 \pm 1{ }^{\circ} \mathrm{C}, 70 \%$ relative humidity and $14 \mathrm{~h}$ light $/ 10 \mathrm{~h}$ dark photoperiod. Larvae were reared on an artificial diet mainly made from wheat germ and soybean powder [39].

A colony of adult $O$. sinensis nematodes was collected from a wheat field in Shangcai, Henan, China. These nematodes were maintained in the laboratory until infective juveniles were obtained. Approximately one thousand juveniles were applied for genomic DNA extraction using a bacterial DNA kit (OMEGA, USA). The primers (Additional file 6: Table S4) were designed to amplify the V4-V5 region of $16 \mathrm{~S}$ rRNA. Then PCR reactions were performed with $30 \mathrm{ng}$ of genomic DNA as a template.

Fifteen juvenile nematodes were used to infect a fourth-instar larva of $H$. armigera for $3 \mathrm{~h}$, as described previously [14]. After successful parasitization by $O$. sinensis, $H$. armigera larvae continued to feed on artificial diet until the emergence of fully developed nematodes. The number of dead larvae was recorded every day. Infected larvae, which died before the emergence of 
fully developed nematodes, were dissected to confirm whether the juveniles succeeded in penetrating. Survival curves were created and analyzed using GraphPad software. Log-rank (Mantel-Cox) test was performed to calculate statistical significance.

\section{Phagocytosis assay}

Fluorescein isothiocyanate (FITC; Sigma) labeling of E. coli was conducted at $37^{\circ} \mathrm{C}$ for $1 \mathrm{~h}$. Samples were washed five times with PBS, and FITC-labeled bacteria were resuspended in PBS to a final concentration of $2 \times 10^{8}$ cells $/ \mathrm{ml}$. Phagocytosis analyses were performed in triplicate, as described previously [40], with minor modifications. Briefly, hemocytes were collected from parasitized and non-parasitized larvae at various time points and suspended in PBS. Subsequently, PBS containing FITC-labeled bacteria was added to the PBS containing hemocytes. After incubation for $1 \mathrm{~h}$, aliquots of the mixture were dispensed onto glass slides, and hemocytes were allowed to settle down for $30 \mathrm{~min}$. Hemocytes were then fixed with $4 \%$ formaldehyde for $10 \mathrm{~min}$, washed and then observed under a fluorescence microscope. The phagocytic rate was calculated as follows:

Phagocytic rate $(\%)=[$ Number of bacteria-ingesting hemocytes

/Total number of hemocytes] $\times 100$

\section{Measurement of antibacterial activity}

Hemolymph was collected from parasitized and non-parasitized larvae at various time points, and diluted 3-fold in sterile anticoagulant buffer. After centrifugation at $1000 \mathrm{rpm}$ for $10 \mathrm{~min}$, cell-free plasma was obtained, and antibacterial activity was determined as described previously [15]. Briefly, $90 \mu \mathrm{l}$ of plasma was mixed with $10 \mu \mathrm{l}$ of $E$. coli suspension. After incubation for $1 \mathrm{~h}$ at room temperature, the plasma-bacteria mixture was plated onto lysogeny broth agar plates and incubated at $37^{\circ} \mathrm{C}$ overnight. Subsequently, the number of colony forming units (CFU) was counted in each plate.

\section{Measurement of PO activity}

To evaluate the effect of parasitism by $O$. sinensis on PO activity of $H$. armigera, plasma was collected from parasitized and non-parasitized larvae at various time points. PO activity was measured as described previously [41]. Briefly, plasma $(50 \mu \mathrm{l})$ was incubated with $50 \mu \mathrm{l}$ trypsin $(2 \mathrm{mg} / \mathrm{ml})$ for $20 \mathrm{~min}$ at room temperature, followed by addition of $50 \mu \mathrm{l}$ substrate solution containing dopamine $(3 \mathrm{mg} / \mathrm{ml})$. Initial absorbance was measured at $490 \mathrm{~nm}$, and one unit of PO activity was defined as the amount of enzyme yielding an increase of 0.001 absorbance units per min.

\section{RNA extraction, DGE library preparation and RNA-Seq analysis}

To prepare DGE libraries, three biological replicates of FP and FC samples were collected at 1, 3 and 5 dpp. Total RNA was extracted from these samples using TRIzol Reagent (Invitrogen), and used with oligo dT magnetic beads to enrich mRNAs. The mRNA samples were then fragmented into short sequences and reverse transcribed using N6 random primer. Subsequently, the resulting double-stranded complimentary DNA was end-repaired to generate blunt ends and ligated with two blunt end adaptors. Following PCR amplification, the PCR products were denatured, and single-stranded DNA was cyclized using splint oligo. The prepared libraries were subjected to SE50 sequencing at the Beijing Genomics Institute.

\section{Mapping reads to the reference unigenes and analysis of DETs}

The high number of unknown bases, adaptor sequences and low quality reads were filtered from raw sequence reads to generate clean reads. Clean reads were then mapped onto reference sequences of $H$. armigera fat body transcriptome generated previously [15] using Bowtie2 [42]. To eliminate the influence of gene length and sequencing discrepancy, gene transcripts were quantified as FPKM values for comparing expression levels of DETs among samples. Correction for false positive and false negative errors were performed using false discovery rates (FDR), with FDR $\leq 0.001$ as the default threshold to judge the significance of gene expression differences. Definition of divergence probability of each transcript differentially expressing was following the formula described previously [43]. Values of $\mid \log 2$ fold-change $\mid \geq 1$ and divergence probability $\geq 0.8$ were used as cut-off thresholds for identifying DETs between the different experimental conditions, based on a non-parametric algorithm NOISeq-sim [44].

\section{Validation of gene expression using qRT-PCR}

Results of RNA-Seq analysis were validated using qRT-PCR. Total RNA (2 $\mu \mathrm{g})$ prepared for RNA-Seq was used for the synthesis of first-strand cDNA. Subsequently, qRT-PCR was conducted with TransStart Top Green qPCR SuperMix (TransGen Bio-tech, Beijing, China) using a CF $\times 96$ system (Bio-Rad, Hercules, CA, USA). A total of eight differentially expressed genes, including PRRs (CTL4, CTL7), AMPs (gloverin, cecropin 3), SPs (SP4, azurocidin-like SP), and mucins (mucin protein, mucin 4), were selected for qRT-PCR analyses. The expression level of each gene was normalized relative to that of the reference gene $\beta$-actin using the $2^{-\Delta C T}$ method $\left(\Delta \mathrm{C}_{\mathrm{T}}=\mathrm{C}_{\mathrm{T}[\text { test gene }]}-\mathrm{C}_{\mathrm{T}[\beta \text {-actin }]}\right)$. Gene-specific primers used for qRT-PCR analysis are listed in Additional file 6: Table S4. 


\section{Additional files}

Additional file 1: Figure S1. PCR analyses confirming the association of O. sinensis with bacteria. M, DL2000 DNA marker. Lane 1, PCR amplicon of V4-V5 region of 165 rRNA. (TIF $38 \mathrm{~kb}$ )

Additional file 2: Table S1. Statistics of DGE library sequencing and reads mapping. (XLS $20 \mathrm{~kb}$ )

Additional file 3: Figure S2. Heatmap indicating the square of correlation value from three biological replicates. The correlation values were assessed by using the Pearson method. (TIF $476 \mathrm{~kb}$ )

Additional file 4: Table S2. Transcripts differentially expressed between the fat body of parasitized and non-parasitized larva. (XLS $3723 \mathrm{~kb}$ )

Additional file 5: Table S3. Immune-related DETs between the fat body of parasitized and non-parasitized larva. (XLS 191 kb)

Additional file 6: Table S4. List of primers used. (XLS 21 kb)

\section{Abbreviations}

AMPs: antimicrobial peptides; CFU: colony forming units; CTL: C-type lectin; DETs: differentially expressed transcripts; DGE: digital gene expression; dpp: d post parasitism; EPNs: entomopathogenic nematodes; FC: fat body of nonparasitized; FDR: false discovery rates; FITC: Fluorescein isothiocyanate; FP: fat body of parasitized; FPKM: fragments per kilobase of transcript per million mapped fragments; GNBP: Gram negative binding protein; HCS: head capsule slippage; PAE: prophenoloxidase activating enzyme; PGRP: peptidoglycan recognition protein; PO: phenoloxidase; PPO: prophenoloxidase; PRRs: pattern recognition receptors; qRTPCR: quantitative real-time PCR; SPs: serine proteases

\section{Acknowledgements}

Not applicable.

\section{Funding}

This work was supported by Grants from the National Natural Science Foundation of China (Grant Nos. 31872301 and 31772220), and Natural Science Foundation of Hubei Province (Grant No. 2016CFB560). The funding bodies played no roles in the study design and data collection, analysis, interpretation of data and in writing the manuscript.

\section{Availability of data and materials}

The data sets have been deposited at the NCBI Sequence Read Archive (SRA) database (http://www.ncbi.nlm.nih.gov/sra/) under the accession number PRJNA497980. Other data supporting the results are available in the additional files.

\section{Authors' contributions}

The study was conceived and designed by JLW. GXW and XSL participated in the design and coordination of the work. GJW and XRZ performed the infection, RNA extraction and qRT-PCR. GJW and WWW conducted phagocytosis assays, measurement of antibacterial and PO activities. JLW, GJW and XSL analyzed the RNA-seq data and prepared the manuscript. All authors have read and approved the final version of the manuscript.

\section{Ethics approval and consent to participate}

Not applicable.

\section{Consent for publication}

Not applicable.

\section{Competing interests}

The authors declare that they have no competing interests.

\section{Publisher's Note}

Springer Nature remains neutral with regard to jurisdictional claims in published maps and institutional affiliations.
Received: 4 October 2018 Accepted: 18 February 2019

Published online: 27 February 2019

\section{References}

1. Lemaitre B, Hoffmann J. The host defense of Drosophila melanogaster. Annu Rev Immunol. 2007:25:697-743.

2. Williams MJ. Drosophila hemopoiesis and cellular immunity. J Immunol. 2007;178(8):4711-6.

3. Castillo JC, Reynolds SE, Eleftherianos I. Insect immune responses to nematode parasites. Trends Parasitol. 2011;27(12):537-47.

4. Dillman AR, Sternberg PW. Entomopathogenic nematodes. Curr Biol. 2012; 22(11):R430-1.

5. Gaugler R, Lewis E, Stuart RJ. Ecology in the service of biological control: the case of entomopathogenic nematodes. Oecologia. 1997;109(4):483-9.

6. Forst S, Dowds B, Boemare N, Stackebrandt E. Xenorhabdus and Photorhabdus spp.: bugs that kill bugs. Annu Rev Microbiol. 1997;51:47-72

7. Owuama Cl. Entomopathogenic symbiotic bacteria, Xenorhabdus and Photorhabdus of nematodes. World J Microbiol Biotechnol. 2001;17:505-15.

8. Eleftherianos I, Joyce S, Ffrench-Constant RH, Clarke DJ, Reynolds SE. Probing the tri-trophic interaction between insects, nematodes and Photorhabdus. Parasitology. 2010;137(11):1695-706

9. Hallem EA, Rengarajan M, Ciche TA, Sternberg PW. Nematodes, bacteria, and flies: a tripartite model for nematode parasitism. Curr Biol. 2007;17(10):898-904.

10. Castillo JC, Shokal U, Eleftherianos I. Immune gene transcription in Drosophila adult flies infected by entomopathogenic nematodes and their mutualistic bacteria. J Insect Physiol. 2013:59(2):179-85.

11. Aliota MT, Fuchs JF, Mayhew GF, Chen CC, Christensen BM. Mosquito transcriptome changes and filarial worm resistance in Armigeres subalbatus. BMC Genomics. 2007:8:463.

12. Wang $\mathrm{G}$, Chen $\mathrm{Q}$, Chen $\mathrm{G}$. In vitro cultivation of the entomogenous nematode Ovomermis sinensis. Acta Zool Sin. 2001:47(2):235-9.

13. Li Q, Sun Y, Wang G, Liu X. Effects of the mermithid nematode Ovomermis sinensis on the hemocytes of its host Helicoverpa armigera. J Insect Physiol. 2009;55(1):47-50.

14. Wang $P$, Zhuo XR, Tang L, Liu XS, Wang YF, Wang GX, Yu XQ, Wang JL. Ctype lectin interacting with $\beta$-integrin enhances hemocytic encapsulation in the cotton bollworm, Helicoverpa armigera. Insect Biochem Mol Biol. 2017 ; 86:29-40

15. Wang JL, Chen L, Tang L, Zhao HB, Liu XS, Wang YF. 20-hydroxyecdysone transcriptionally regulates humoral immunity in the fat body of Helicoverpa armigera. Insect Mol Biol. 2014;23(6):842-56.

16. Dunphy GB, Thurston GS. Insect immunity. In: Gaugler R, Kaya HK, editors. Entomopathogenic nematodes in biological control, vol. 30. Boca Raton, FL: CRC press; 1990. p. 326.

17. Muttis E, Micieli MV, Urrutia MI, García JJ. Transmission of a pathogenic virus (Iridoviridae) of Culex pipiens larvae mediated by the mermithid Strelkovimermis spiculatus (Nematoda). J Invertebr Pathol. 2015;129:40-4.

18. Noda H, Miyoshi T, Zhang Q, Watanabe K, Deng K, Hoshizaki S. Wolbachia infection shared among planthoppers (Homoptera: Delphacidae) and their endoparasite (Strepsiptera: Elenchidae): a probable case of interspecies transmission. Mol Ecol. 2001;10(8):2101-6.

19. Kim CH, Shin YP, Noh MY, Jo YH, Han YS, Seong YS, Lee $\mathbb{H}_{\text {. }}$. An insect multigand recognition protein functions as an opsonin for the phagocytosis of microorganisms. J Biol Chem. 2010;285(33):25243-50.

20. Cheng $Y$, Lin Z, Wang JM, Xing LS, Xiong GH, Zou Z. CTL14, a recognition receptor induced in late stage larvae, modulates anti-fungal immunity in cotton bollworm Helicoverpa armigera. Dev Comp Immunol. 2018;84:142-52.

21. Li L, Li YP, Song CX, Xiao M, Wang JL, Liu XS. Identification and functional characterization of a peptidoglycan recognition protein from the cotton bollworm, Helicoverpa armigera. Arch Insect Biochem Physiol. 2014;86(4):240-58.

22. Brivio MF, Mastore $M$, Nappi AJ. A pathogenic parasite interferes with phagocytosis of insect immunocompetent cells. Dev Comp Immunol. 2010; 34(9):991-8.

23. Wang $X$, Fuchs JF, Infanger LC, Rocheleau TA, Hillyer JF, Chen CC, Christensen BM. Mosquito innate immunity: involvement of beta 1,3-glucan recognition protein in melanotic encapsulation immune responses in Armigeres subalbatus. Mol Biochem Parasitol. 2005;139(1):65-73.

24. Fang Q, Wang F, Gatehouse JA, Gatehouse AM, Chen XX, Hu C, Ye GY. Venom of parasitoid, Pteromalus puparum, suppresses host, Pieris rapae, immune promotion by decreasing host C-type lectin gene expression. PLoS One. 2011;6(10):e26888. 
25. Wang L, Fang Q, Qian C, Wang F, Yu XQ, Ye G. Inhibition of host cell encapsulation through inhibiting immune gene expression by the parasitic wasp venom calreticulin. Insect Biochem Mol Biol. 2013;43(10):936-46.

26. Ferrandon D, Imler JL, Hetru C, Hoffmann JA. The Drosophila systemic immune response: sensing and signalling during bacterial and fungal infections. Nat Rev Immunol. 2007;7(11):862-74.

27. Magalhaes T, Oliveira IF, Melo-Santos MA, Oliveira CM, Lima CA, Ayres CF. Expression of defensin, cecropin, and transferrin in Aedes aegypti (Diptera: Culicidae) infected with Wuchereria bancrofti (Spirurida: Onchocercidae), and the abnormal development of nematodes in the mosquito. Exp Parasitol. 2008;120(4):364-71.

28. Erickson SM, Xi Z, Mayhew GF, Ramirez JL, Aliota MT, Christensen BM, Dimopoulos $G$. Mosquito infection responses to developing filarial worms. PLoS Negl Trop Dis. 2009;3(10):e529.

29. Chalk R, Townson H, Ham PJ. Brugia pahangi: the effects of cecropins on microfilariae in vitro and in Aedes aegypti. Exp Parasitol. 1995;80(3):401-6.

30. Jarosz J. Active resistance of entomophagous rhabditid Heterorhabditis bacteriophora to insect immunity. Parasitology. 1998; 117 (Pt 3):201-208.

31. Korayem AM, Fabbri M, Takahashi K, Scherfer C, Lindgren M, Schmidt O, Ueda R, Dushay MS, Theopold U. A Drosophila salivary gland mucin is also expressed in immune tissues: evidence for a function in coagulation and the entrapment of bacteria. Insect Biochem Mol Biol. 2004;34(12):1297-304.

32. Tang $H$, Kambris Z, Lemaitre $B$, Hashimoto $C$. Two proteases defining a melanization cascade in the immune system of Drosophila. J Biol Chem. 2006;281(38):28097-104.

33. Zou Z, Shin SW, Alvarez KS, Kokoza V, Raikhel AS. Distinct melanization pathways in the mosquito Aedes aegypti. Immunity. 2010;32(1):41-53.

34. Lee SY, Kwon TH, Hyun JH, Choi JS, Kawabata SI, Iwanaga S, Lee BL. In vitro activation of pro-phenol-oxidase by two kinds of pro-phenol-oxidaseactivating factors isolated from hemolymph of coleopteran, Holotrichia diomphalia larvae. Eur J Biochem. 1998;254(1):50-7.

35. Volz J, Müller HM, Zdanowicz A, Kafatos FC, Osta MA. A genetic module regulates the melanisation response of Anopheles to Plasmodium. Cell Microbiol. 2006;8(9):1392-405.

36. Chu Y, Hong F, Liu Q, An C. Serine protease SP105 activates prophenoloxidase in Asian corn borer melanization, and is regulated by serpin-3. Sci Rep. 2017;7:45256

37. Balasubramanian N, Hao YJ, Toubarro D, Nascimento G, Simões N. Purification, biochemical and molecular analysis of a chymotrypsin protease with prophenoloxidase suppression activity from the entomopathogenic nematode Steinernema carpocapsae. Int J Parasitol. 2009;39(9):975-84.

38. Balasubramanian N, Toubarro D, Simões N. Biochemical study and in vitro insect immune suppression by a trypsin-like secreted protease from the nematode Steinernema carpocapsae. Parasite Immunol. 2010;32(3):165-75.

39. Zhao XF, An XM, Wang JX, Dong DJ, Du XJ, Sueda S, Kondo H. Expression of the Helicoverpa cathepsin B-like proteinase during embryonic development. Arch Insect Biochem Physiol. 2005;58(1):39-46.

40. Li J, Song CX, Li YP, Li L, Wei XH, Wang JL, Liu XS. Rab3 is involved in cellular immune responses of the cotton bollworm, Helicoverpa armigera. Dev Comp Immunol. 2015;50(2):78-86.

41. Qiao C, Li J, Wei XH, Wang JL, Wang YF, Liu XS. SRP gene is required for Helicoverpa armigera prophenoloxidase activation and nodulation response. Dev Comp Immunol. 2014;44(1):94-9.

42. Langmead B, Trapnell C, Pop M, Salzberg SL. Ultrafast and memory-efficient alignment of short DNA sequences to the human genome. Genome Biol. 2009;10(3):R25

43. Zhang Y, Wang P, Xia H, Zhao C, Hou L, Li C, Gao C, Wang X, Zhao S. Comparative transcriptome analysis of basal and zygote-located tip regions of peanut ovaries provides insight into the mechanism of light regulation in peanut embryo and pod development. BMC Genomics. 2016;17(1):606.

44. Tarazona S, García-Alcalde F, Dopazo J, Ferrer A, Conesa A. Differential expression in RNA-seq: a matter of depth. Genome Res. 2011;21(12):2213-23.

Ready to submit your research? Choose BMC and benefit from:

- fast, convenient online submission

- thorough peer review by experienced researchers in your field

- rapid publication on acceptance

- support for research data, including large and complex data types

- gold Open Access which fosters wider collaboration and increased citations

- maximum visibility for your research: over $100 \mathrm{M}$ website views per year

At BMC, research is always in progress.

Learn more biomedcentral.com/submissions 